ema: Metalurgia do pó

\title{
AVALIAÇÃO DO CARBETO DE NIÓBIO E COBRE ATRAVÉS DE DIFRAÇÃO DE RAIOS-X E REFINAMENTO RIETVELD*
}

\author{
Maria Veronilda Macedo Souto ${ }^{1}$ \\ Maria José Santos Lima² \\ Uilame Umbelino Gomes ${ }^{3}$ \\ Carlson Pereira de Souza 4
}

\section{Resumo}

Este trabalho teve como objetivo a síntese do carbeto de nióbio e cobre $\mathrm{Cu}-\mathrm{NbC}$ nanoestruturados e análise por DRX e refinamento Rietveld. A síntese foi realizada através de reação heterogênea a baixa temperatura $\left(1.000^{\circ} \mathrm{C}\right)$ e curto tempo de reação ( 2 horas). O carbeto de nióbio foi produzido com $5 \%$ de cobre. $O$ material obtido foi caracterizados através dos ensaios de Difração de Raios- X (DRX) e Refinamento Rietveld. A partir das análises de DRX e do refinamento Reitiveld, observou-se a formação do carbeto de nióbio e cobre puro com estrutura cúbica e tamanho de cristalito de $34,97 \mathrm{~nm}$ para o $\mathrm{Cu}-\mathrm{NbC}$, concluindo que o composto é nanomêtrico.

Palavras-chave: Carbeto de nióbio; Cobre; Reação heterogênea; Metalogia do pó.

\section{EVALUATION OF NIOBIUM CARBIDE BY X-RAY DIFFRACTION AND RIETVELD REFINEMENT}

\section{Abstrat}

This work aimed at the synthesis of nanostructured niobium and copper carbide $\mathrm{Cu}-\mathrm{NbC}$ and XRD analysis and Rietveld refinement. The synthesis was performed by heterogeneous reaction at low temperature $\left(1,000^{\circ} \mathrm{C}\right)$ and short reaction time ( 2 hours). The niobium carbide was produced with 5\% copper. The material obtained was characterized by X-ray Diffraction (XRD) and Rietveld refinement. From the XRD and refinement Rietveld, we observed the formation of pure niobium and copper carbide with cubic structure and crystallite size of $34.97 \mathrm{~nm}$ for $\mathrm{Cu}-\mathrm{NbC}$, concluding that the compound is nanometric.

Keywords: Niobium carbide; Copper; Heterogeneous reaction; Powder metalogy.

1 Licenciatura, Química, doutoranda, estudante, Departamento de Ciências e Engenharia de Materiais da Universidade Federal do Rio Grande do Norte, Universidade Federal do Rio Grande do Norte - UFRN, Natal, RN, Brasil.

2 Licenciatura/Química, doutoranda, estudante, Departamento de Ciências e Engenharia de Materiais da Universidade Federal do Rio Grande do Norte, Universidade Federal do Rio Grande do Norte - UFRN. Natal, RN, Brasil.

3 Engenheiro, Engenharia Química, Doutor, Professor, Departamento de Engenharia química da Universidade Federal do Rio Grande do Norte, Universidade Federal do Rio Grande do Norte UFRN. Natal, RN, Brasil.

4 Licenciatura/Físico, Doutor, Professor, Departamento de Ciências e Engenharia de Materiais da Universidade Federal do Rio Grande do Norte, Universidade Federal do Rio Grande do Norte UFRN. Natal, RN, Brasil.

\footnotetext{
* Contribuição técnica ao $69^{\circ}$ Congresso Anual da ABM - Internacional e ao 14ํㅡㄹ ENEMET - Encontro Nacional de Estudantes de Engenharia Metalúrgica, de Materiais e de Minas, 21 a 25 de julho de 2014, São Paulo, SP, Brasil.
} 


\section{INTRODUÇÃO}

O carbeto de nióbio $(\mathrm{NbC})$ apresenta várias propriedades, tais como, elevada dureza, alto ponto de fusão $\left(3610^{\circ} \mathrm{C}\right)$, elevada condutividade térmica, grande estabilidade química e até resistência à radiação. Por estes motivos vem se destacando na indústria mecânica, na área química e de microeletrônica [1].

Foi preparado pela primeira vez pela reação de $\mathrm{K}_{2} \mathrm{O}_{3} \mathrm{Nb}_{2} \mathrm{O}_{5}$ com carbono.

Industrialmente o $\mathrm{NbC}$ é produzido aquecendo uma mistura do metal com carbono ou uma mistura do óxido de metal com carbono, em ambos os casos, a reação de carburização acontece pela difusão em estado sólido. Estes processos requerem altas temperaturas entre $1600^{\circ} \mathrm{C}$ e $1800^{\circ} \mathrm{C}$ e longos tempos de reação [2].

Por viabilidade econômica e industrial, busca-se a produção de carbetos em temperaturas mais baixas $\left(1000^{\circ} \mathrm{C}\right)$ e tempos de reação mais curtos [1]. Os carbetos podem ser produzidos por processos homogêneos, porém a utilização de reações heterogêneas, apresenta-se bastante eficaz neste processo de síntese de carbetos, pois este tipo de reação acontece intra e/ou extrapartícula, favorecendo o fenômeno de transferência de massa e de difusão, o que não ocorre em reações homogêneas [3].

Atualmente o carbeto de nióbio tem recebido grande atenção devido a sua aplicação em vários setores da indústria, pois se apresenta como um material de reforço muito promissor em aplicações industriais, como por exemplo, em revestimento de reatores nucleares e catalisadores para a indústria química [4]. É usado frequentemente em aços microligados devido ao seu produto de solubilidade extremamente baixa na austenita, o menor de todos os carbonetos metálicos refratários [1].

Existem na literatura, alguns estudos sobre a adiçao de um ligante ao carbeto, como por exemplo, adição de cobalto como ligante em carbeto de tungstênio, esse processo modifica as propriedades desses matériais, podendo citar a dureza, a maleabilidade, dentre outras.

O cobre é elemento químico que pode ser usado como ligante do carbeto, pois apresenta excelentes propriedades químicas e físicas.

Além disso, a dopagem com $\mathrm{Cu}$ aumenta a atividade catalítica em processos de oxidação de hidrogênio, os íons de $\mathrm{Cu}^{2+}$ modificam as propriedades magnéticas exibidas pelos compostos, assim como o mecanismo de transporte elétrico, o efeito de magnetorresistência colossal e o efeito magnetocalórico [5].

Assim, este trabalho teve objetivo principal a sintetização de carbeto de nióbio e cobre e análise por DRX e Refinamento Rietveld.

\section{MATERIAIS E MÉTODOS}

O carbeto de nióbio foi sintetizado com $5 \%$ de cobre em baixa temperatura a partir do precursor tris(oxalato)oxiniobato de amônio hidratado macerado em almofariz. $\mathrm{O}$ hidrogênio $\left(\mathrm{H}_{2}\right)$ e o metano $\left(\mathrm{CH}_{4}\right)$ foram utilizados como gases redutores e fonte de carbono respectivamente. As reações entre o precursor e a mistura de metano e hidrogênio foram efetuadas em um forno resistivo, composto de um reator de leito fixo de alumina. Para acomodar a amostra de precursor no reator de leito fixo foi utilizada uma barquinha de alumina, utilizando para todos os ensaios uma massa de 2 gramas, que foi introduzida no tubo de alumina até a parte central do forno. Após o fechamento do tubo, o mesmo foi lavado por alguns minutos com argônio a fim de

\footnotetext{
* Contribuição técnica ao $69^{\circ}$ Congresso Anual da ABM - Internacional e ao 14ํㅡㄹ ENEMET - Encontro Nacional de Estudantes de Engenharia Metalúrgica, de Materiais e de Minas, 21 a 25 de julho de 2014, São Paulo, SP, Brasil.
} 


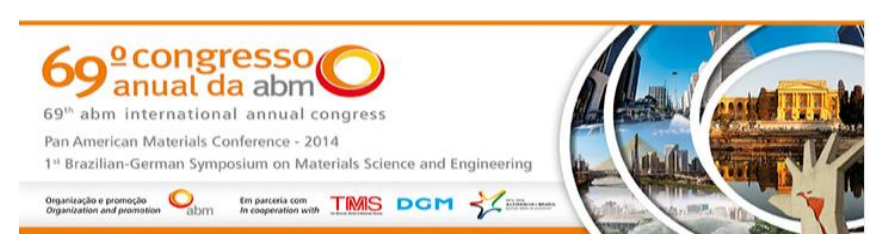

eliminar todo o oxigênio atmosférico presente. Em seguida foram ajustados os fluxos dos gases reagentes (metano e hidrogênio) e a mistura gasosa foi circulada através do reator. Para a determinação das etapas de reações envolvidas e propor um mecanismo reacional que represente todo o processo reacional da síntese do $\mathrm{Cu}-\mathrm{NbC}$, as reações de decomposição-redução-carbonetação foram feitas na temperatura de $1000^{\circ} \mathrm{C}$ e no tempo de isoterma de 120 minutos. Foi utilizado fluxo de hidrogênio e de metano, durante a reação. Ao final da reação de decomposiçãoredução-carbonetação, o fluxo de gases reagentes foi trocado por um fluxo de argônio o qual foi mantido até a temperatura ambiente, quando as amostras foram retiradas.

\section{RESULTADO E DISCUSSÕES}

Os resultados obtidos por difratograma de Raios-X, após o processo de síntese, indicaram a formação do carbeto de nióbio com cobre. Os ângulos de difração apresentaram intensidades que indicam que 0 produto produzido experimentalmente, $\mathrm{Cu}-\mathrm{NbC}$, é puro. O refinamento Rietveld indicou a formação do carbeto de nióbio com cobre de estrutura cúbica de face centrada (CFC) e permitiu determinar os parâmetros cristalográficos das amostras, o desvio em relação aos dados da literatura e o tamanho médio das partículas.

A Figura 1 é referente ao difratograma de raios-x do carbeto de nióbio com $5 \%$ de cobre, o qual indica a formação de picos característicos do carbeto de nióbio( $\mathrm{NbC})$, que estão identificados com $\left({ }^{*}\right)$. Os padrões experimentais de difração de raios- $X$ também mostram a presença dos picos característicos do cobre nos ângulos de 43,27 e 50,48, respectivamente, o que indica a presença do cobre e formação do composto de interesse $(\mathrm{Cu}-\mathrm{NbC})$. Esse resultado confirma que o processo de síntese via gás-sólido adotado para a realização desse trabalho é bastante eficaz para a produção do compósito de nióbio nanoestruturado.

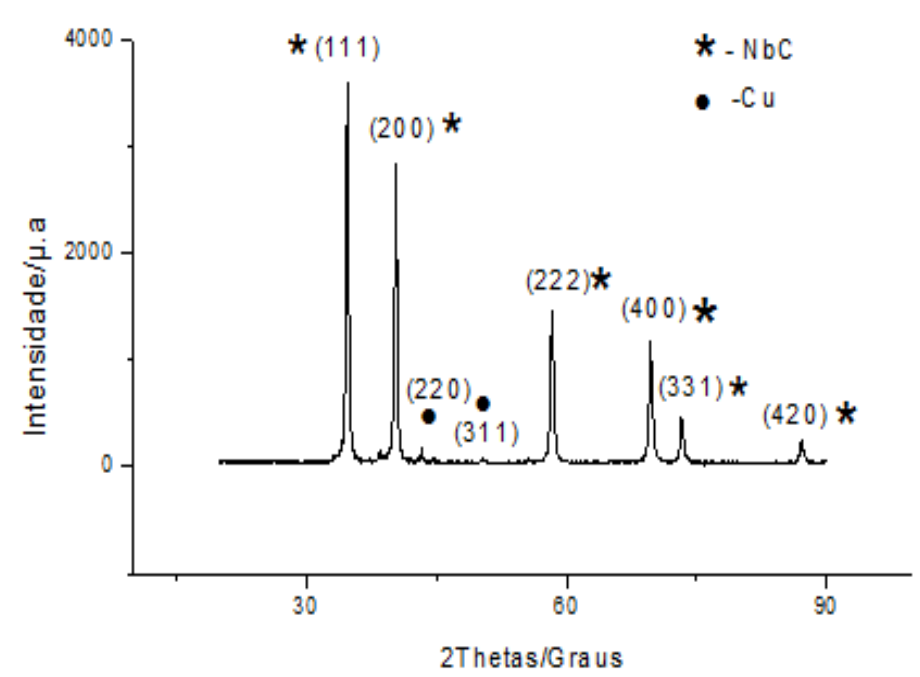

Figura 1. Difratograma de Raios-X $5 \% \mathrm{Cu}-\mathrm{NbC}$ sintetizado.

Na Figura 2 é apresentado os padrões de difração de Raios-X do carbeto de nióbio com $5 \%$ de cobre refinado pelo método Rietveld. O refinamento para as amostras revelaram a formação de fase cristalina na forma cúbica e com os seguintes parâmetros cristalográficos: $a=4,4705663(\AA), b=4,4705663(\AA)$ e c = 4,4705663( $\AA$ ). O refinamento também relevou os valores de Alpha $\left(^{\circ}\right)=90,0000$,

\footnotetext{
* Contribuição técnica ao $69^{\circ}$ Congresso Anual da ABM - Internacional e ao 14ํㅡㄹ ENEMET - Encontro Nacional de Estudantes de Engenharia Metalúrgica, de Materiais e de Minas, 21 a 25 de julho de 2014, São Paulo, SP, Brasil.
} 
Beta $\left(^{\circ}\right)=90,0000$, Gama $\left(^{\circ}\right)=90,0000$, o volume da célula $=4,4470566$, obteve um desvio igual a $S=1,298$ e tamanho médio de cristalito igual $35,9 \mathrm{~nm}$.

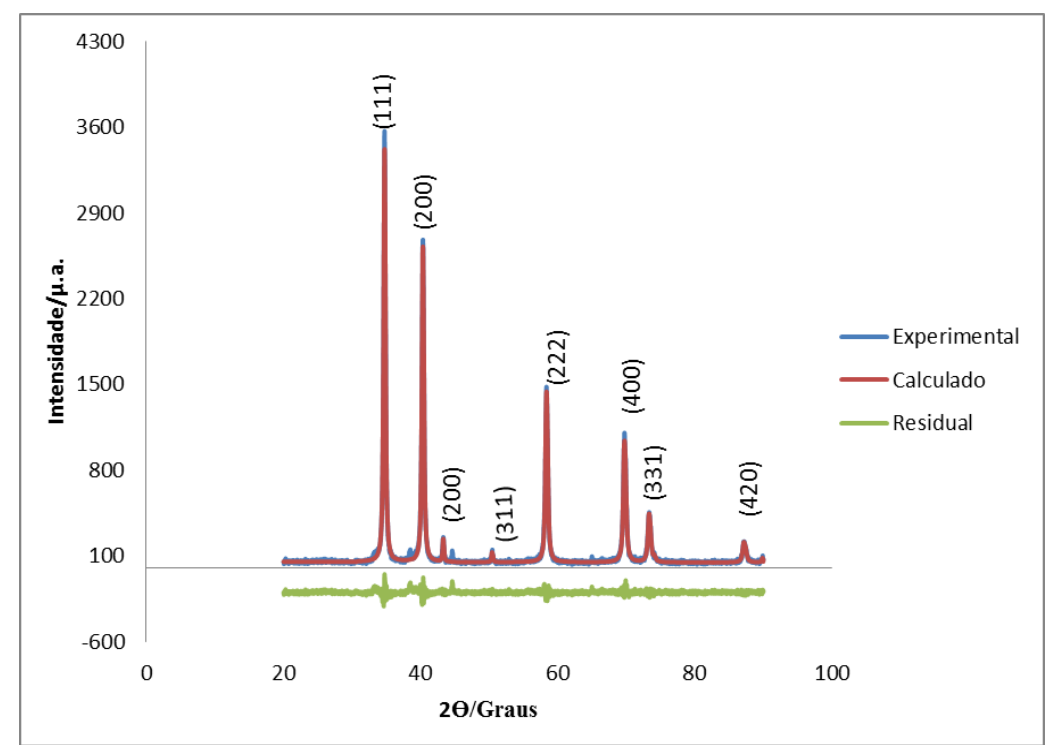

Figura 2. Espectro de Difração de Raios-X refinado pelo Método Rietveld.

A Tabela 1 abaixo estão apresentados alguns dos principais resultados referentes ao refinamento realizado para o carbeto de nióbio com $5 \%$ de cobre.

Tabela 1. Dados do refinamento do $5 \% \mathrm{Cu}-\mathrm{NbC}$ pelo Software Maud

\begin{tabular}{lccccc}
\hline \multicolumn{1}{c}{ Fases } & $\begin{array}{c}\text { Densidade } \\
\left(\mathrm{g} / \mathrm{cm}^{3}\right)\end{array}$ & $\begin{array}{c}\text { Volume de } \\
\text { Célula }(\AA)\end{array}$ & $\begin{array}{c}\text { Tamanho de } \\
\text { cristalito }(\mathrm{nm})\end{array}$ & \% em massa & Estrutura \\
\hline $\begin{array}{l}\text { NbC-43493- } \\
\text { ICSD }\end{array}$ & 8,91 & 4,4470566 & 35,9 & 95,61 & CFC \\
\hline $\begin{array}{l}\text { Cu-94449- } \\
\text { ICSD }\end{array}$ & 7,81 & 3,61 & 98,07 & 4,39 & \\
\hline
\end{tabular}

Para confirmar o tamanho do cristalito e a microdeformação do Cu-NbC, também, foram realizadas outras duas medidas, uma utilizando o Método de Scherrer e a outra - Método de Williamson-Hall. Os resultados obtidos dessas medidas são apresentados nas Tabelas 2 e 3 . Esses resultados comprovaram que o $\mathrm{Cu}-\mathrm{NbC}$ produzido apresenta um tamanho de cristalito na ordem de nanômetros e os valores de microdeformação são desprezíveis.

Tabela 2. Tamanho médio de cristalito obtido pelo Método de Scherrer

\begin{tabular}{|c|c|c|c|c|c|c|}
\hline Material & & & $5 \% \mathrm{Cu}-$ & $\mathrm{bC}$ & & \\
\hline Posição(2Ө) & 34,71 & 40,303 & 58,333 & 69,713 & 73,300 & 89,300 \\
\hline $\mathrm{TC}(\mathrm{nm})$ & 30,33 & 30,397 & 27,428 & 27,649 & 27,108 & 28,355 \\
\hline $\mathrm{Tm}(\mathrm{nm})$ & & & & & & 28,546 \\
\hline
\end{tabular}

Legenda: $T m$ = tamanho médio de cristalito, Tc= tamanho de cristalito

Tabela 3. Tamanho médio de cristalito obtido pelo Método de Williamson-Hall

\begin{tabular}{c|c|c}
\hline Material & $\operatorname{Tm}(\mathrm{nm})$ & Md $(\%)$ \\
\hline $\mathrm{CuNbC}$ & 34,97 & 0,000550
\end{tabular}

Legenda: $T m$ = Tamanho médio de Cristalito; $M d=$ Microdeformação

* Contribuição técnica ao $69^{\circ}$ Congresso Anual da ABM - Internacional e ao 14ํㅡㄹ ENEMET - Encontro Nacional de Estudantes de Engenharia Metalúrgica, de Materiais e de Minas, 21 a 25 de julho de 2014, São Paulo, SP, Brasil. 
4 CONCLUSÕES

Os estudos realizados neste trabalho revelaram que o método de síntese, através da reação heterogênea, gás-sólido, é satisfatório para a produção de Cu-NbC, pois os resultados de DRX apresentados revelaram a formação de fase pura do carbeto e a incorporação do cobre no composto. $\mathrm{E}$ que de acordo com o refinamento rietveld $\mathrm{o}$ carbeto de nióbio com $5 \%$ de cobre apresentou o tamanho de cristalito de ordem nanométrica.

\section{Agradecimentos}

Programa de Pós-Graduação em Ciências e Engenharia de Materiais- PPGCEM; Universidade Federal do Rio Grande do Norte- UFRN;

Laboratório de Materiais Cerâmicos e Metais Especiais - LMCME; Laboratório de Materiais Nanoestruturados e Reatores Cataliticos - LAMNRC; Coordenação de Aperfeiçoamento de Pessoal de Nível Superior-CAPES

\section{REFERÊNCIAS}

1 Medeiros FFP. Síntese de carbetos de tungstênio e nióbio a baixa temperatura, através de reação gás-sólido em reator de leito fixo [tese Doutorado em Engenharia Química].

Natal: Centro de Tecnologia, Departamento de Engenharia Química, Universidade Federal do Rio Grande do Norte; 2002.

2 Furukava F. Sinterização de aço inoxidável reforçado com partículas nonométricas dispersas de carbeto de nióbio [tese de doutorado]. Natal: Ciências e Engenharia de Materiais, UFRN; 2007.

3 Souto MVM. Síntese e caracterização de $\mathrm{CuNb}_{2} \mathrm{O}_{6}$ e $\mathrm{CuNbC}$ através de reação sólidosólido e gás-sólido a baixa temperatura [Dissertação de Mestrado]. Natal: Programa de Pós-Graduação em Ciência e Engenharia de Materiais, Universidade Federal do Rio Grande do Norte; 2013.

4 Roth RW, Lishner B. Fracture behavior of composites based on Al2O3-TiC. Jornal of materials Science. 1980;15:875-885.

5 Vasconcelos BR, Morais AMV, Lopes FWB, Souza CP. Estudo da variação da concetração de cu na/no $\mathrm{CuNb}_{2} \mathrm{O}_{6}$ sintetizado a partir de reação sólido-sólido. In: Anais do XVIII Congresso Brasileiro de Engenharia Química COBEG; 2010; Paraná, Brasil. Paraná: COBEG; 2010.

* Contribuição técnica ao 69ํㅡㄹ Congresso Anual da ABM - Internacional e ao 14ํㅡㄹ ENEMET - Encontro Nacional de Estudantes de Engenharia Metalúrgica, de Materiais e de Minas, 21 a 25 de julho de 2014, São Paulo, SP, Brasil. 